\title{
Historia de la robótica: de Arquitas de Tarento al Robot da Vinci.
} (Parte II)

\author{
Sánchez-Martín FM, Jiménez Schlegl P*, Millán Rodríguez F, Salvador-Bayarri J, \\ Monllau Font V, Palou Redorta J, Villavicencio Mavrich H. \\ Servicio de Urología. Fundació Puigvert. Barcelona. *Institut de Robótica Industrial (IRI). Univ. Politécnica de \\ Cataluña. CSIC. \\ Actas Urol Esp. 2007; 31(3):185-196
}

"Los brazos de acero cromado de Robbie abrazaban cariñosamente a la chiquilla..."

Isaac Asimov, "Yo robot". 1950

\section{RESUMEN}

HISTORIA DE LA ROBÓTICA: DE ARQUITAS DE TARENTO AL ROBOT DA VINCI. (PARTE II)

La cirugía robótica es ya una realidad. Revisar la historia de la robótica antigua (ver parte I) y moderna es importante para conocer cómo funcionan los nuevos robots. La fabricación de máquinas que imitan al ser humano se ha mantenido desde hace más de 4000 años. En la robótica clásica destacaron inventores como Arquitas de Tarento (hacia 400 a.C.), Heron de Alejandría, Hsieh-Fec, AlJazari, Bacon, Turriano, Leonardo da Vinci, Vaucanson o von Kempelen (ver parte I). En 1942 Asimov publica las tres leyes de la robótica. Con el desarrollo de la mecánica, la electrónica y la informática en el siglo XX se han desarrollado robots capaces de realizar de forma autónoma tareas de gran complejidad. En 1985 el robot PUMA 560 fue utilizado para introducir una aguja en el cerebro. A partir de ahí se desarrollan robots cirujanos como World First, Robodoc, Gaspar o Acrobot, Zeus, AESOP, Probot o PAKI-RCP. En el año 2000 la FDA aprueba el da Vinci Surgical System (Intuitive Surgical Inc, Sunnyvale, CA, USA) un sofisticado robot asistente del cirujano. Procedimientos urológicos como la prostatectomía, cistectomía o la nefrectomía son realizadas con el robot da Vinci, situando a la urología como una de las especialidades más idóneas para la cirugía robótica.

Palabras clave: Robótica. Urología Robótica. Historia de la Robótica. Historia de la Medicina. Arquitas.

\section{ABSTRACT}

HISTORY OF ROBOTICS: FROM ARCHYTAS OF TARENTUM UNTIL DA VINCI ROBOT. (PART II)

Robotic surgery is a reality. In order to to understand how new robots work is interesting to know the history of ancient (see part i) and modern robotics. The desire to design automatic machines imitating humans continued for more than 4000 years. Archytas of Tarentum (at around 400 a.C.), Heron of Alexandria, Hsieh-Fec, Al-Jazari, Bacon, Turriano, Leonardo da Vinci, Vaucanson o von Kempelen were robot inventors. At 1942 Asimov published the three robotics laws. Mechanics, electronics and informatics advances at $\mathrm{XX}^{\text {th }}$ century developed robots to be able to do very complex self governing works. At 1985 the robot PUMA 560 was employed to introduce a needle inside the brain. Later on, they were designed surgical robots like World First, Robodoc, Gaspar o Acrobot, Zeus, AESOP, Probot o PAKI-RCP. At 2000 the FDA approved the da Vinci Surgical System (Intuitive Surgical Inc, Sunnyvale, CA, USA), a very sophisticated robot to assist surgeons. Currently urological procedures like prostatectomy, cystectomy and nephrectomy are performed with the da Vinci, so urology has become a very suitable speciality to robotic surgery.

Keywords: Robotics. Robotics in Urology. History of Medicine. History of Robotics. Archytas. 
$\mathrm{E}$ n los últimos años la robótica se ha ido introduciendo en medicina, siendo la cirugía el terreno donde se han producido los avances más interesantes. La construcción de máquinas que imitan las tareas humanas se remonta a la antigüedad. En la parte I se ha revisado los antecedentes de la robótica (desde la antigüedad hasta el siglo XIX) ${ }^{1}$. En esta parte II se hace un repaso cronológico a los hechos más relevantes en la robótica moderna y la inteligencia artificial (siglos XX y XXI).

Desde los años 80 los robots se han ido incorporando a las tareas quirúrgicas hasta alcanzar cotas de gran virtuosismo, como las logradas con el proyecto da Vinci Surgical System (Intuitive Surgical, Sunnyvale, CA, USA). La cirugía con este robot asistente hace posibles intervenciones de gran envergadura a partir de las órdenes manuales de un cirujano que trabaja a distancia sobre una consola. El futuro de la cirugía estará muy ligado a los robots, en las que el ser humano ha volcado su inventiva desde la antigüedad.

\section{Historia de la robótica moderna}

En el primer tercio del siglo XX se inicia el desarrollo de la ingeniería en sus diferentes ramas (mecánica, electrónica, informática, telecomunicaciones) que van a permitir la construcción de robots modernos. La lista de acontecimientos científicos y técnicos que tienen que ver con la robótica no se limita a la ingeniería sino que involucra a las matemáticas y la física teórica. Incluso las formulaciones de Lagrange, Newton y Euler, efectuadas en el clasicismo, son fundamentales para desarrollar después las ecuaciones que explican la dinámica y la inteligencia de los robots actuales. Los avances en computación de las últimas décadas son el impulso definitivo que permite desarrollar máquinas muy cercanas al ideal de automatismo y autonomía que siempre persiguieron los constructores de robots.

Asimov utilizó por primera vez el término "robótica" en los relatos cortos reunidos en su libro I Robot (Yo robot) ${ }^{2}$ publicado en 1950 (el relato se había publicado previamente en 1942 en un magazine de ciencia ficción) ${ }^{3}$. En el relato titulado "Runaround", ambientado en el año 2056, se postulan las tres leyes de la robótica (Tabla 1). Su
Tabla 1

Las tres leyes de la robótica según Asimov

1. Un robot no debe dañar a un ser humano ni, por su pasividad, dejar que un ser humano sufra daño.

2. Un robot debe obedecer las órdenes que le son dadas por un ser humano, excepto cuando estas órdenes están en oposición con la primera Ley.

3. Un robot debe proteger su propia existencia, hasta donde esta protección no esté en conflicto con la primera o segunda ley.

ámbito novelístico no ha impedido que sigan vigentes hasta la actualidad, cuanto menos como referente teórico. Asimov consideró necesario añadir una cuarta ley, antepuesta a las demás, la número cero, que afirma que un robot no debe actuar simplemente para satisfacer intereses individuales, sino que sus acciones deben preservar el beneficio común de toda la humanidad. La aportación literaria de Asimov se produjo en un momento de auge de la robótica moderna, durante la primera mitad del siglo XX.

En 1938 H Roselund y W Pollard, de la compañía Devilviss, construyen el primer brazo articulado (o manipulador) para pintura al spray ${ }^{4}$ lo que representó una nueva forma de entender la producción industrial al incorporar robots a las cadenas de producción, donde hasta entonces sólo habían operado trabajadores humanos. En 1939 se presentan diversas novedades en robótica popular, como el robot humanoide Elektro, fabricado por Westinghouse, y el perro mecánico Sparko, atracciones en la feria mundial de Nueva York celebrada aquel año. Se trataba de máquinas que realizaban movimientos simples, de escasa articulación, con rutinas mecánicas repetitivas. Se adivinan en esa época dos de las grandes ramas de la robótica moderna: la industrial y la de ocio.

Los avances en inteligencia artificial son paralelos a los de la robótica. En los años 40 Huffman, aporta sus investigaciones algoritmos de combinatoria realimentada que permiten desarrollar sistemas secuenciales provistos de una cierta capacidad de memoria útil para construir una serie de funciones progresivas. La formalización del tratamiento de los automatismos se basa en el álgebra de Boole y en la teoría de 
autómatas finitos. Entre 1943 y 1946 H Goldstine, P Eckert y $\boldsymbol{J}$ Mauchly, del Ballistic Research Laboratory (BRL), construyen la primera computadora digital de la historia a la que denominan ENIAC (Electronic Numerical Integration and Calculador) con la que la computación contemporánea se consolida y se introduce en las empresas. Durante la segunda guerra mundial se desarrolla un robot con dos brazos teleoperados para manipular explosivos. Este tipo de máquinas manipuladoras no se consideran robots en sentido estricto pues responden a las órdenes de un teleoperador humano y, un robot, ejecuta sus acciones siguiendo las instrucciones de un programa. El reciente advenimiento de telemanipuladores "inteligentes" en medicina hace que la definición de robot se haya ampliado a este tipo de máquinas. En 1947 DS Halder, de la compañía automovilística Ford de Detroit, acuña el término "automatización" y pone en marcha, una estrategia para ir sustituyendo al ser humano de muchas de las tareas del proceso de fabricación de automóviles (Fig. 1). En 1948 G Walter, de la Bristol University, presentó el primer robot autónomo electrónico.

En 1950 ocurren importantes sucesos para el devenir de la robótica: AM Touring publica "Computing Machinery and Intelligence" y propone una prueba (test o máquina de Touring), en forma de entidad matemática abstracta, que demuestra la existencia de problemas computacionales irresolubles que ninguna máquina es capaz de solventar ${ }^{5}$. Se puede afirmar que un programa de ordenador no llegará nunca a ser tan inteligente como un ser humano y que un robot no

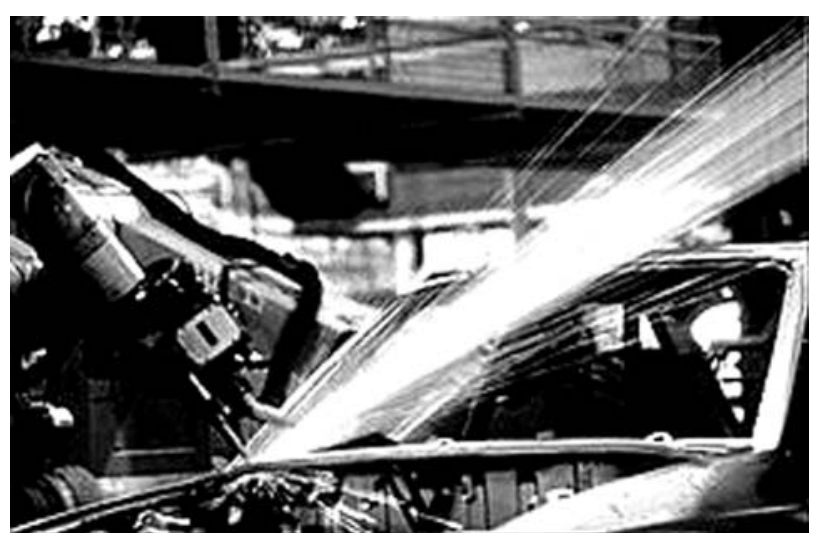

FIGURA 1. Robot, tipo brazo manipulador, trabajando en la industria automovilistica. podrá suplir al ser humano de forma completa. Y aparece otro libro importante: "El uso humano de los seres humanos: cibernética y sociedad"6, de $\boldsymbol{N}$ Wiener donde se acuña por primera vez el término cibernética y se discuten los conceptos de comunicación y control entre humanos y máquinas, que venían madurando desde las dos décadas anteriores, incorporando las ideas barajadas por pensadores como J Clerk-Maxwell, $\mathbf{S}$ Odobleja o L Couffignal. En 1951 W Shockley inventa el transistor y hace posible una nueva generación de computadoras mucho más rápidas y pequeñas. Los montajes se miniaturizan y las máquinas resultantes son más manejables, fáciles de construir y baratas. A partir de todos estos avances y del interés de los industriales por automatizar las tareas de producción, la robótica va adquiriendo un gran desarrollo. Los robots industriales suponen la superación de los conceptos en los que se basaban las máquinas previas: concepto máquina-herramienta, automatización rígida, manipuladores secuenciales ${ }^{7}$. Los hitos históricos de la robótica industrial son de gran importancia para entender los de otros campos como el doméstico, el militar, el aeroespacial o el quirúrgico. En el Instituto Tecnológico de Massachussets (MIT) se desarrolla en 1952 una línea de investigación sobre control numérico que culmina con la definición del lenguaje de programación de piezas tipo APT (Automatically Programmed Tooling), publicado en 1961, básico para la automatización programable. En 1953 se construye en Inglaterra el primer robot móvil autónomo de la historia, llamado ELSIE (ElectroLight-Sensitive Internal-External), que podía seguir una fuente de luz utilizando un sistema mecánico realimentado sin incorporar inteligencia adicional ni tener dependencia de órdenes externas. En 1955 J Denavit y RS Hartenberg utilizan el álgebra matricial para describir y representar la geometría espacial de los elementos de un robot. GC Devol, uno de los pioneros de la robótica industrial, patenta en 1956 un controlador que registraba señales eléctricas por medio de magnetos que accionan un dispositivo mecánico, logrando una máquina flexible, adaptable al entorno y fácil de manejar ${ }^{7}$. A partir de Devol es cuando puede denominarse robot a una máquina. El carácter de reprogramabilidad la 
distingue de los autómatas. Los aspectos relacionados con el control son fundamentales para la asignación de tareas a un robot.

G Goertz (1954) y D Bergsland (1958) investigan sobre manipuladores mecánicos a control remoto (teleoperación) para el manejo de material radiactivo. Se trata de un sistema amo-esclavo (master-slave), en que el robot reproduce de forma fiel y en tiempo real los movimientos que un operador le transmite a distancia. A Newell y H Simon postulan en 1956 "The Logic Theorist", el primer sistema experto, que resuelve algunos problemas matemáticos y permite el diseño de algoritmos funcionales imprescindibles en robótica. Ese mismo año $\boldsymbol{J}$ McCarthy, M Minsky, $\boldsymbol{N}$ Rochester y $\boldsymbol{C}$ Shannon organizan una reunión conocida como "The Dartmouth Summer Research Project on Artificial Intelligence" donde el término inteligencia artificial de Wiener se oficializa y se marcan unos objetivos de investigación, cumplidos sólo parcialmente. La pretensión de desarrollar un programa que solucionara cualquier tipo de problema -el GPS General Problem Solver- no ha tenido continuidad. Hoy se opta por programas más especializados, con atribuciones específicas, conservando una estructura jerárquica con diferentes niveles de abstracción.

En los años 50 el acoplamiento mecánico comenzó a sustituirse por los sistemas eléctricos e hidráulicos, gracias al desarrollo de los triodos, dando lugar a manipuladores como el Handyman de General Electric Co. y Minotaur de General Mills ${ }^{8}$. En 1957 Plannet Corporation patenta el primer robot industrial controlado por interruptores de fin de carrera. Ese año el inventor británico $\boldsymbol{C W}$ Kenward obtiene la primera patente de la historia para un robot industrial y CY Lee define en 1959 los programas de decisiones binarias para el desarrollo de automatismos combinatorios, una herramienta alternativa a la lógica Booleriana útil para la descripción de automatismos combinatorios, ya que el álgebra de Boole (Fig. 2) no puede modelar la dinámica de los sistemas secuenciales. Ese año Edford Associates proponen un sistema de control denominado Controlador Digital Modular a la General Motors Co. ${ }^{7}$ que dará paso a los sistemas de control automático

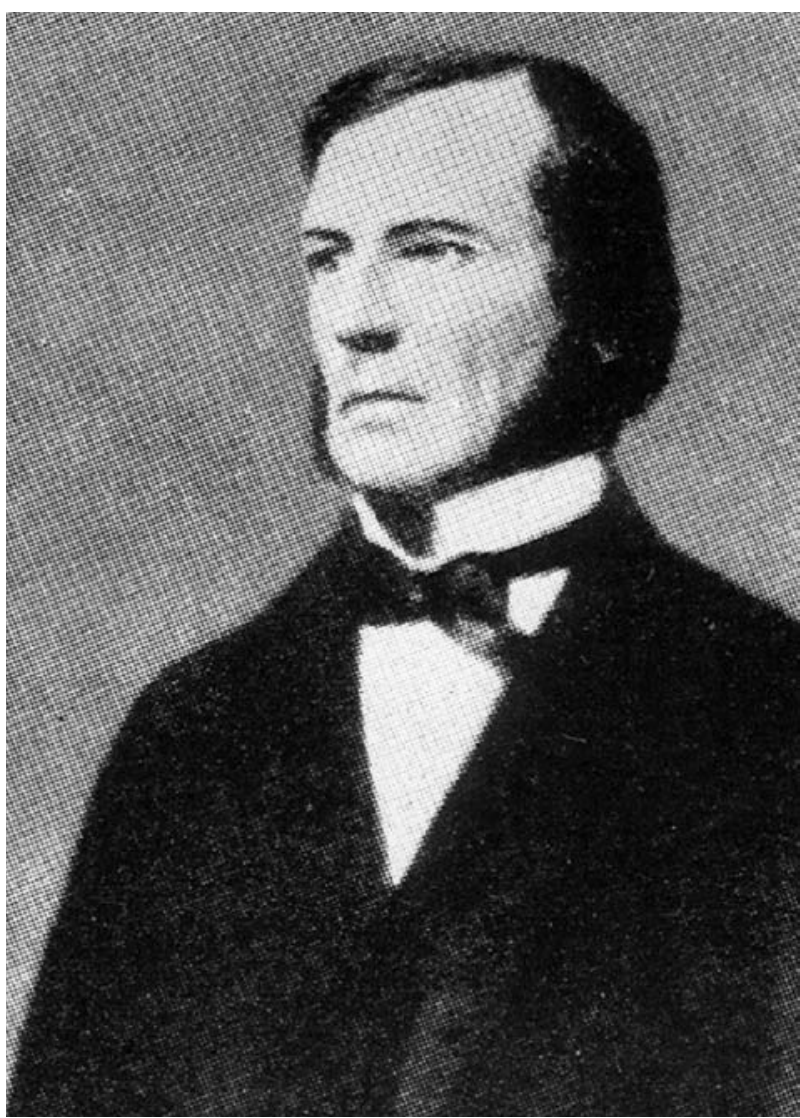

FIGURA 2. El matemático George Boole.

actuales, consistentes en máquinas de estado secuencial y procesadores centrales con desplazamiento de bits. En 1958 es fundada por Engelberger la empresa Unimation -hoy absorbida por Westinhouse- que en 1960 pone en marcha el primer robot tipo "Unimate", basado en la transferencia de artículos programados, siguiendo los principios de Devol postulados en 1954. El robot disponía de un manipulador basado en control numérico y se movía por transmisión hidráulica. El control numérico permite evaluar las acciones de una máquinaherramienta mediante dígitos, según lo describió originalmente $\boldsymbol{J}$ Parsons, que concibió el empleo de tarjetas perforadas con datos posicionales para la puesta en marcha de resortes mecánicos.

El lenguaje de programación tipo APT (Automatically Programmed Tooling) fue desarrollado en el MIT entre 1952 y 1961. En 1960 Devol vendió sus patentes a Condec, que comienza a fabricar el Unimate en su subsidiaria Unimation ${ }^{4}$. 
Tres años después se inicia la implementación de robots en la industria automovilística en Europa, a la que Japón se suma en 1968.

En el año 1962 ocurren diversos acontecimientos de gran importancia para la robótica. HA Enst publica un trabajo sobre sensores táctiles $\mathrm{MH}-1$ aplicados a una mano robotizada de tipo ANL 8, dotada de 6 grados de libertad y de un procesador TX-O que revoluciona el sector e inicia el desarrollo de los sensores y la retroalimentación, demostrando la conducta adaptativa de un robot por primera vez en la historia. El robot apilaba bloques en un entorno no estructurado valiéndose de sensores volumétricos y de peso, a los que después se añadió una cámara de TV (proyecto MAC) para obtener más precisión y autonomía. Por su parte $\boldsymbol{R}$ Tomovic y $\boldsymbol{G}$ Boni desarrollan una mano con sensores de presión que proporciona una señal de realimentación de entrada al motor para iniciar uno de los dos modelos de aprehensión disponibles en función del peso del objeto ${ }^{8}$. Al mismo tiempo CA Petri desarrolla un sistema de redes para el diseño y análisis de automatismos secuenciales y concurrentes, en que son tan importantes los componentes mecánicos y eléctricos como los sistemas de control (computación y comunicación), que se aplica en el análisis y modelado de sistemas, no sólo en el campo de la automática, sino también en el de la informática y las comunicaciones. Por su parte General Motors Co. instala el primer robot Unimate en su planta automovilística para asumir la soldadura en troquel ${ }^{4}$.

En 1963 las empresas American Machine y Foundry Co (AMF) introducen el robot comercial Versatran $^{9}$ y ese año se desarrollan otros brazos manipuladores como el Roehampton y el Edinburg $^{8}$. En 1966 la empresa Trallfa consigue importantes avances en automatización con un robot de pintura por pulverización; y en 1968 es construido en el Standford Research Institute (SRI) el robot móvil Shakey, provisto de sensores táctiles y una cámara de visión. J McCarthy, del Stanford Artificial Inteligente Laboratory, publica el desarrollo de una computadora con "manos, ojos y oídos" (manipuladores, cámaras de TV y micrófonos) para reconocimiento de mensajes verbales, visualización de bloques sobre una superficie y capacidad de manipulación según las instrucciones programadas. Ese mismo año, en el mismo centro se confecciona el WAVE, el primer lenguaje de programación para robots, siguiendo criterios de computación, y DL Pieper contribuye a la solución del problema cinemática de manipuladores controlados por computadora. En 1966, el gurú de la inteligencia artificial $\boldsymbol{M}$ Minsky reflexiona sobre la gran dificultad para desarrollar un modelo evolutivo a partir de la genética binaria, con el que las máquinas vayan adquiriendo facultades de locomoción, habla, reconocimiento facial, comprensión y traducción de lenguajes, destreza manual, equilibrio y orientación espacial.

En 1968 aparece Shakey, del Stanford Research Institute, provisto de múltiples sensores y de medios para desplazarse por el suelo y de control remoto por radio. $\mathrm{Al}$ año siguiente son diseñados los brazos robóticas Stanford y Boston, sobre los que se basarán las investigaciones en los siguientes años; y General Electric Co. desarrolla, para la US Army, un vehículo motorizado (camión) experimental para conducción a control remoto y con funciones autónomas. En los años setenta la NASA inicia un programa de cooperación con el Jet Propulsión Laboratory (JPL) para desarrollar plataformas con posibilidad de exploración de terrenos agrestes, produciendo el Mars-Rover, un vehículo robotizado que disponía de un brazo Stanford y multitud de unidades sensoras. Por su parte la empresa Kawasaki se erige como la pionera en robótica en Japón al negociar en 1968 una licencia con la americana Unimation. El ingeniero de la compañía Yaskawa, $\boldsymbol{T}$ Mori, acuña el término "mecatrónica" que integra, el conjunto de mecanismos de control automático imprescindibles para el desarrollo de cualquier máquina inteligente ${ }^{10}$ (Fig. 3).

Hasta 1970 todos los robots funcionaban con actuadores hidráulicos pero ese año el SRI construye un manipulador con 6 grados de libertad accionado por motores eléctricos ${ }^{4}$. En 1970 la Universidad de Stanford crea el Stanford Cart, un robot que sigue "visualmente", de forma autónoma, líneas en el suelo, con opción a control remoto por radio. En 1970 la agencia espacial rusa consigue un alunizaje por control remoto y explo- 


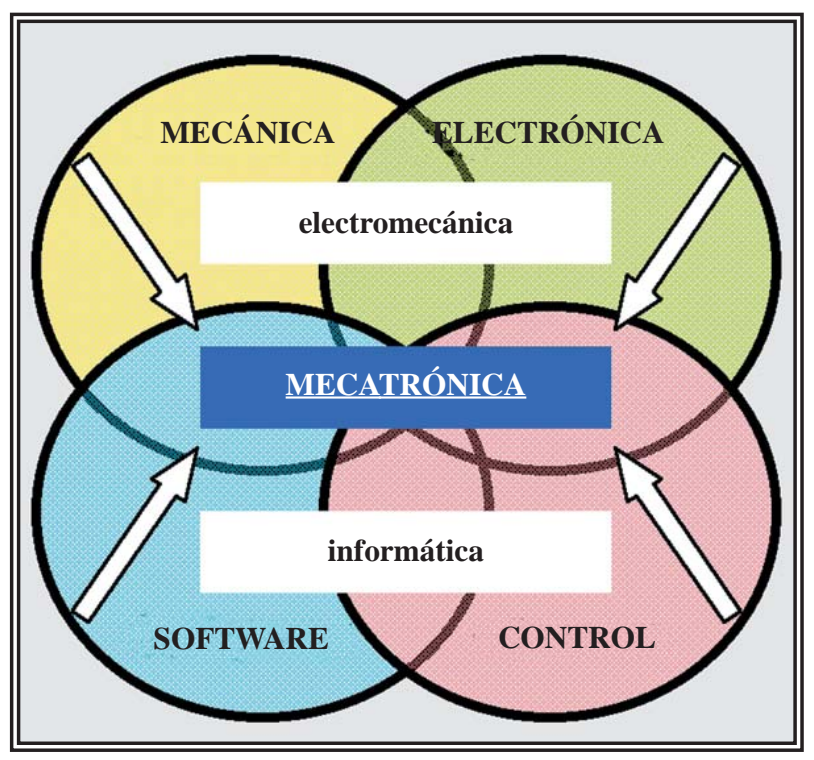

FIGURA 3. Diagrama mecatrónico de construcción de máquinas inteligentes.

ran la superficie lunar con un vehículo robotizado dotado de sensores, efectores y una unidad de telecomunicaciones, llamado Lunokhod $\mathrm{I}^{11}$. En 1971 ME Kahn y B Roth resuelven la dinámica de un brazo para movimiento curvo (loop) utilizando un control de tiempo mínimo tipo bangbang ${ }^{12}$.

Las funciones de comunicación comenzaron a integrarse en los autómatas a partir del año 1973, con el Modbus de Modicon como primer bus de comunicaciones. La posibilidad de comunicación entre mecanismos y sistemas de control se realiza mediante las Power Line Comunications (PLC), que pueden intercambiar informaciones con otros dispositivos y en 1974 aparece el lenguaje AL, que facilita aún más la comunicación. V Scheinman y $B$ Simano unificaron ambos sistemas (VAL) para la empresa comercial Unimation.

En 1974 RC Bolles y RP Paul utilizan realimentación visual y de fuerzas demuestran que un brazo robótico Stanford es capaz de montar una bomba de agua ${ }^{13}$, hecho considerado un hito en lo referente a autonomía y precisión. Ese año Cincinnati Milacron presenta el T3 o "The Tomorow Tool", el primer robot asistido por computadora capaz de levantar objetos de 50 kilos de peso y seguir objetos en una línea de montaje. Por su parte Kawasaki inicia la experiencia en soldadura por arco robotizada en su fábrica de motocicletas; y la firma ASEA fabrica el robot Irb6 que disponía de accionamiento totalmente eléctrico. H Inoue, del MIT, trabaja en 1974 en inteligencia artificial de realimentación de fuerzas, usando técnicas aeronáuticas (aterrizaje) para reproducirlas en un esquema de montaje de piezas de alta precisión. En 1978 DE Whitney y JL Nevins, del Draper laboratory, investigan el control por fuerza y posición (técnicas sensoriales) y desarrollan el Remote Center Compliance destinado a la última generación de manipulador consiguiendo perfeccionar el montaje de piezas sueltas. En 1975 la aplicación de microprocesadores en robótica revoluciona este sector, logrando que mejore el tamaño y el precio de los robots. Al mismo tiempo la empresa Olivetti utiliza el robot Sigma en operaciones de montaje; y la Air Force ICAM (Integrated Computer- Aided Manufacturing) integra los robots T3 de Cincinati Milacron en la industria aeronáutica. PM Will y DD Grossman desarrollan entonces un prototipo para montaje de motores en $\mathrm{IBM}^{14}$, y AK Bejczy, del JPL, adapta un brazo Stanford para un proyecto espacial ${ }^{15}$. El 1979 la empresa Three Mile Island estimula la aplicación de robots (soldadura, fontanería) en la industria nuclear. Entre 1975 y 80 el parque de robots aumenta un 25\% a expensas de la industria del automóvil ${ }^{7}$. En 1976 el Viking norteamericano aterriza en Marte y pone en marcha un brazo manipulador capaz de recoger muestras del suelo y tomar imágenes en detalle. El robot PUMA (Programmable Universal Machine for Assembly) de Unimation, desarrollado en 1978, comienza a trabajar para General Motors Co. en tareas de montaje. Fue usado también para investigación, por las posibilidades que ofrecía su lenguaje de programación, VAL y VAL-II.

A partir de 1980 se investiga en controles informáticos y sensores, lo que potencia la construcción de robots inteligentes capaces de adaptarse al ambiente (interacción) y tomar decisiones ${ }^{7}$. En 1980 un sistema robótico de captación de recipientes fue objeto de demostración en la Universidad de Rhode Island. Usando la visión de máquina el sistema era capaz de controlar piezas en orientaciones espaciales aleatorias. El robot SCARA (Selective Compliance Arm for Robotic 
Assembly) es desarrollado por la Universidad de Yamanashi y explotado comercialmente para la industria en 1981. Ese mismo año se desarrolla en la Universidad Carnegie-Mellon un robot de impulsión directa que utiliza motores eléctricos en las articulaciones, evitando la distorsión de las transmisiones mecánicas convencionales. En 1982 IBM introduce el robot de montaje industrial RS-1 que utiliza un brazo constituido por 3 dispositivos de deslizamiento. El trasbordador Columbia es el primero en utilizar ese año el primer robot teleoperador (brazo) en el espacio.

Mientras tanto Westinghouse Corp. pone en marcha un proyecto piloto en 1983 para una línea de montaje automatizada flexible con el empleo de robots, basado en el sistema de montaje programable adaptable (APAS). En 1984 comienzan a funcionar los Robots 8 que responden a programas gráficos interactivos, preprogramados en una computadora personal y luego cargados en el robot. Ese mismo año D Lenat desarrolla el proyecto Cyc para dotar a los robots de órganos sensibles $^{16}$.

En 1985 un vehículo submarino guiado a control remoto participa en las tareas de rescate del avión de la Air Indian, siniestrado frente a las costas de Irlanda, y pocos meses después otro robot similar descubre los restos del Titanic a 4.000 metros de profundidad. El 21 de julio de 1984 ocurre la primera muerte de la historia relacionada con un robot que aplasta contra una barra de protección a un trabajador en la ciudad de Jackson (Michigan).

Cuando en 1985 RF Curl, HW Kroto y RE Smalley (premios Nóbel de química en 1996) descubren la "buckminsterfullernes" (buckyballs), esferoides de carbono de un nanómetro de diámetro, sientan las bases de la nanotecnología y dan inicio a la investigación en dispositivos automáticos destinados a la fabricación de nanites o nanorobots, que hoy se halla aún en fase de desarrollo ${ }^{17}$. Una de las pretensiones es emplear robots miniaturizados, micro o incluso nanorrobots, en medicina, insertándolos en el torrente sanguíneo para el tratamiento local de tumores. En 1986 Honda inicia su programa de I+D en robótica con la consigna de que un robot "debe coexistir y cooperar con los seres humanos, haciendo lo que una persona no puede hacer...para beneficiar a la sociedad".

Los investigadores del MIT, $\boldsymbol{R}$ Brooks y $\boldsymbol{A M}$ Flynn, publican en 1989 el artículo "Fast, cheap and out of control: A Robot Invasion of the Solar System", que cambia la filosofia de la construcción de robots. Se trata de no hacer pocos robots, grandes y caros, sino muchos robots, pequeños y baratos. Al mismo tiempo el Mobile Robots Group del MIT desarrolla un robot hexápodo llamado Genghis.

En 1993 D Cliff, I Harvey y P Husbands de la Universidad de Sussex introducen los robots evolucionarios, que presentan las secuencias de control del robot dentro de una estructura genómica, imitando la evolución que ha experimentado el ser humano. También en 1993 el robot de ocho patas Dante, de la Carnegie-Mellon University intenta descender al interior del Monte Erebus en la Antártida pero, tras 20 pasos, una rotura del arnés precipita el robot al fondo del volcán. Un año después una versión más evolucionada, el Dante II, consigue descender hasta el fondo del cráter estableciendo un hito para los robots móviles que han de trabajar en condiciones extremas.

En 1995 el parque de robots industriales alcanza ya las 700.000 unidades, generando importantes volúmenes de negocio ${ }^{7}$.

En 1996 aparece RoboTuna un robot diseñado por $\boldsymbol{D}$ Barrett, del MIT, para el estudio del movimiento de las especies marinas. C Campbell y $\mathbf{S}$ Wilkinson fabrican el Gastrobot, un robot autopropulsado por dióxido de carbono obtenido de la digestión de materia orgánica. Ese mismo año es liberado en Marte el robot Sojourner, un vehículo de seis ruedas controlado desde La Tierra, dotado de un sistema autónomo de control que compensa los 10 minutos que tarda la orden en ser recibida en Marte. Posteriormente los robots Spirit y Oportunity han explorado con éxito la superficie marciana, sobrepasando ampliamente las previsiones más optimistas ${ }^{18}$.

La robótica doméstica se ha ido consolidando y está apunto de sumarse al consumo de masas. Androides con funciones sociales y de comunicación están en pleno desarrollo, siendo la movilidad uno de los aspectos más importantes. Conseguir que un robot se mantenga en posición 
erecta sobre dos piernas y camine contorneando la cadera como un ser humano ha sido uno de los grandes retos de la robótica moderna. La invención de los hexápodos con plataforma móvil fue un recurso importante para desarrollar modelos de contorneo muy útiles para el diseño de unidades articuladas ${ }^{19}$. Una posibilidad simple y eficaz es asignar la movilidad a 3 ó 4 ruedas, como por ejemplo los semiandroides Minerva ${ }^{20}$, RP6 y Pearl, estos dos últimos destinados a realizar tareas de enfermería y pase de visita hospitalaria. Algunos robots móviles no androides se valen del desplazamiento rodado ya que realizan tareas dinámicas de apoyo al ser humano, como el SR1 (ruedas o correas) $^{21}$ o el PackBot (correas) ${ }^{22}$. Un modelo más antropomórfico se vale de 4 ó 6 patas articuladas, imitando la anatomía de mamíferos e insectos ${ }^{23}$, si bien el objetivo para robots que pretenden convivir en sociedad es desplazarse con dos patas. Los primeros avances en este sentido consiguieron un modelo fijado a un eje central mediante un vástago lateral, llegando finalmente al modelo pseudohumano de posición bipedestante y dinámica motriz, que imita la biomecánica humana, como el correspondiente al KHR-1 de RoboSaby ${ }^{24}$, a partir de la I+D de un modelo de Kondo Kagaku $\mathrm{Co}^{25}$. En 1997 Honda presenta el $\mathrm{P}-3$, un androide de 1,60 m de altura y $130 \mathrm{Kg}$ de peso, capaz de caminar con seguridad sobre dos piernas, imitando la marcha humana gracias a sensores de gravedad, visuales, de detección de ángulos y de aceleración. Entre sus habilidades figuran las de pasear por terreno llano, subir y bajar escaleras, abrir puertas, pulsar interruptores y empujar obstáculos.

Tiger Electronics lanza, en la campaña de Navidad de 1998, el robot Furby, una mascota mecánica que reacciona ante los cambios del entorno y puede comunicarse mediante más de 800 frases en su propio idioma, el "Furbish", o en otras lenguas. La casa danesa LEGO lanza Mindstorms, dentro de su programa Robotics Invention System; y SONY pone en el mercado el Aibo, un robot mascota canina. Al mismo tiempo la Japan Science and Technology Corporation (JST) desarrolla un robot humanoide de 1'80 m. de altura y 80 kilos de peso que camina como un ser humano ${ }^{26}$. En Japón existe un problema de envejecimiento de la población que ha impulsado la investigación en robótica asistencial. En el 2000 Honda lanza su última versión de robot androide, el ASIMO (Advanced Step in Innovative Mobility), de $120 \mathrm{~cm}$ de altura y $43 \mathrm{Kg}$ de peso, disponible en el mercado (Fig. 4) ${ }^{27}$. En los últimos años han sido diseñados otros robots para tareas de limpieza doméstica, como los plafones móviles de iRobot, llamados Scooba y Roomba ${ }^{28}$, de los que ya se han vendido millones de unidades en USA. En el terreno militar destacan robots desarticuladores de explosivos como el PackBot de iRobot $^{29}$, o de vehículos de conducción autónoma como el $\mathrm{r}$-gator ${ }^{30}$. Los androides de tipo dinámico pasivo más modernos como SIGMO, QRIO, ASIMO y Hubo son capaces de caminar, entablar conversación (con evidentes limitaciones) y realizar algunas tareas simples. También están disponibles robots sociales, conocidos por Ludobots, como Wakamaru ${ }^{31}$. La presencia de millones de robots en todo el mundo, en sectores muy diferentes, nada tiene que ver con las 3.500 unidades que funcionaban en $1974^{32}$.

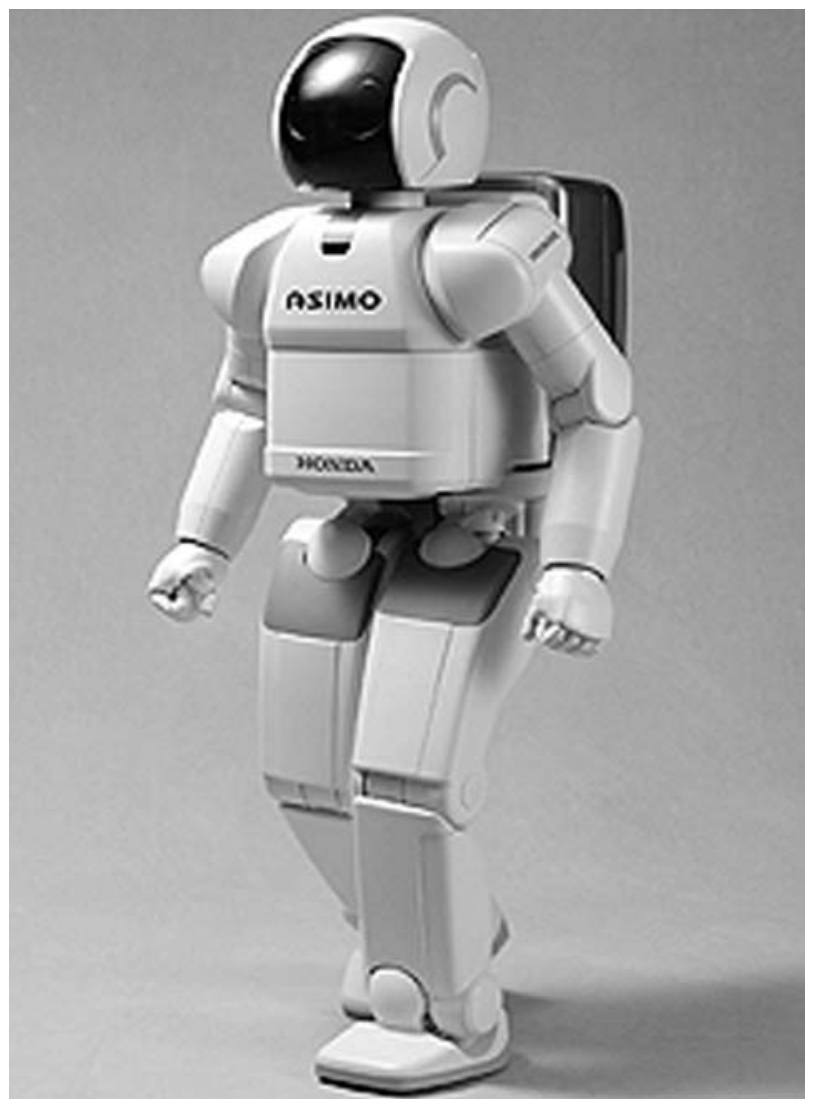

FIGURA 4. El robot humanoide Asimo, de Honda. 


\section{ROBOTS QUIRÚRGICOS}

En 1980 comienzan las primeras experiencias quirúrgicas con robots en neurocirugia y ortopedia $^{33}$ pero no es hasta 1985 cuando el robot PUMA 560 fue utilizado para introducir una aguja en el cerebro mediante guía $\mathrm{TAC}^{34}$. Ese año el Departamento de Ingeniería Mecánica del Imperial College of London (UK) comenzó la investigación en robótica médica orientándola hacia la neurocirugía $^{35}$. En 1986 se inició la investigación para implante robotizado de prótesis de cadera en el Centro de Investigación Thomas J. Watson de IBM y en la Universidad de California ${ }^{36}$. En 1988 el robot inglés "World First" es el primero en el mundo en extraer fragmentos de tejido humano en un quirófano, concretamente en una prostatectomía, por lo que corresponde a la urología este hito de la historia de la robótica ${ }^{37}$. Y Wang funda en 1989 la empresa Computer Motion Inc. (Goleta, CA, USA) especializada en la construcción de robots quirúrgicos, que irá desarrollando los proyectos AESOP, Hermes y Zeus. En 1992 se presenta en Inglaterra Robodoc (Integrated Surgical Systems. UK), el primer asistente mecánico de la cirugía de artroplastia de cadera y rodilla. En esos años la empresa Innovative Medical Machines International, diseña el NeuroMate ${ }^{\mathrm{TM}}$ System, un dispositivo robotizado con aplicaciones en neurocirugia $^{38}$.

Hacia 1993 Computer Motion Inc, comenzó a trabajar en el robot cirujano Zeus, cuyo primer prototipo estuvo disponible en 1995 y fue probado en modelo animal en 1996. La idea original había sido desarrollada en la década de 1980 en un departamento de investigación del ejército norteamericano, con objeto de atender a distancia heridos de guerra. Una vez perfeccionada la máquina fue posible realizar la primera anastomosis entre dos estructuras tubulares en 1998. En el año 2000 se perfeccionaron algunos mecanismos de giro y de articulación. El proyecto AESOP (Automated Endoscopic System for Optical Positioning) se remonta a 1994, con el modelo 1000, que fue el primer robot del mundo aprobado por la FDA. En 1996 la empresa Computer Motion Inc. siguió con las mejoras hasta llegar al AESOP 4000 , disponible hoy día. Se trata de un brazo robotizado inteligente controlado por medio de una tarjeta digitalizada que reconoce la voz de cada cirujano, configurada previamente ${ }^{39}$. El robot es capaz de obedecer órdenes verbales gracias a una interfase de software llamada Hermes $^{40}$. Al final de la década de los 90 se presentaron los resultados con el robot ARTEMIS un robot asistente con imagen bidimensional y dos brazos mecánicos que no ha llegado a tener aplicación clínica ${ }^{41}$.

En 1995 se constituye la empresa Intuitive Surgical Inc (Sunnyvale, CA, USA) con el objeto de desarrollar un proyecto completo de robótica quirúrgica ${ }^{42}$. En 1997 ya había iniciado sus experiencias con un sistema de cirugia robotizada mediante un prototipo llamado Mona, un robot precursor del actual da Vinci, que utilizaba ya un sistema amo-esclavo con consola de mandos y brazos independientes para que un cirujano, situado fuera del campo quirúrgico, comande los brazos robóticos colocados, mediante laparoscopia, en el interior del abdomen del paciente ${ }^{43}$. Con esta técnica se realizaron en Bélgica una colecistectomía (1997) y una cirugía bariátrica (1998), la primera en el mundo realizada por un robot dirigido a distancia ${ }^{44}$.

En 1999 surge el robot quirúrgico más avanzado conocido hasta la actualidad, el da Vinci Surgical System, que en el 2000 obtiene la validación de la FDA para realizar procedimientos de cirugía abdominal vía laparoscópica ${ }^{45}$. Por su parte el robot PROBOT, del Imperial College of London, fue usado en el bienio 1997-98 para realizar operaciones endoscópicas de próstata en Inglaterra ${ }^{46}$, estableciendo un hito en la robótica quirúrgica ya que se trata de un robot autónomo que trabaja después de la preprogramación sobre el caso clínico, una vez obtenidas coordenadas ecográficas.

En septiembre de 2001 se realizó la primera colecistectomía transoceánica con el robot ZEUS, estableciendo un hito en telecirugía. El robot operó a una mujer de 68 años en Estrasburgo (Hospital Civil del Este de Francia), mientras que el equipo de cirujanos controlaba los mandos de la consola desde New York (Mount Sinai Medical Center) a $7.000 \mathrm{Km}$ de distancia ${ }^{47}$. La colaboración entre este centro y el Instituto Francés de Investigación (IRCAD), el Instituto Europeo de Telecirugía (EITS) y las compañías France Telecom 
y Computer Motion hicieron posible este hito de la cirugía, la robótica y la teleasistencia. Este acontecimiento señala que el futuro no sólo apunta hacia la robótica sino también hacia la realización de cirugía entre estaciones de trabajo remotas. En el 2001 la unidad de robótica del Johns Hopkins Medical Institution (Baltimore) presentó los resultados de telecirugía robótica con el robot AESOP 3000 (Brazo asistente) y PAKI-RCM (puncionador renal estereotáxico), en la reunión anual de la AUA con 8 casos de cura de varicocele, 2 biopsias retroperitoneales, una pieloplastia, 3 nefrectomías y 3 accesos percutáneos al riñón ${ }^{48}$, que preludiaban la gran afinidad entre urología, dispositivos robotizados y telecirugia.

Las últimas novedades en robótica quirúrgica vienen de la mano del da Vinci Surgical System (Fig. 5). En los 6 últimos años el robot se ha ido consolidando como el proyecto más avanzado en robótica quirúrgica, después de más de 25 años en desarrollo ${ }^{43}$. Desde 1997 se han ido realizando diversos procedimientos de cirugía abdominal como funduplicatura de Nissen-Rosetti ${ }^{49}$, miotomía de Heller ${ }^{50}$, plicatura gástrica ${ }^{44}$ y anastomosis gastrointestinal tipo Y de Roux, con buenos resultados. La realización de cirugía cardíaca compleja, como el by-pass aorto-coronario ${ }^{51}$ representó asimismo un impulso para la credibilidad del sistema. En junio del 2000, Intuitive Surgical salió a bolsa y en 2003 adquirió a su principal competi-

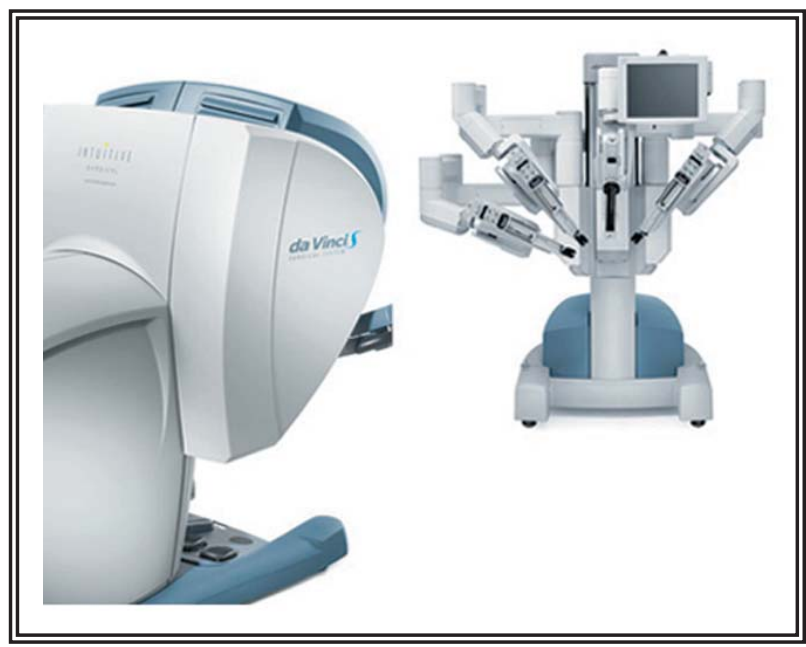

FIGURA 5. Elementos del robot cirujano da Vinci S (consola y soporte con manipuladores). dor, Computer Motion, absorbiendo los proyectos Zeus (similar a da Vinci), AESOP y Hermes, convirtiéndose en el líder mundial de fabricantes de robots quirúrgicos. Las intervenciones con da Vinci han experimentado un aumento constante, pasando de 1.500 procedimientos en el año 2000 a 20.000 en el 2005.

Actualmente existen una diversidad de robots cirujanos que pueden ser clasificados según su nivel de implicación o autonomía: pueden ser robots servo-asistentes (AESOP y otros brazos asistentes), asistentes-coordinadores (Hermes), efectores semiautónomos (Robodoc, Caspar, Acrobot, Probot, PAKI), y la última generación: robots cirujanos de control remoto o telemanejados - teleoperated - (da Vinci).

\section{UROLOGÍA Y ROBOTS QUIRÚRGICOS}

En 1988 se inicia la investigación en robótica urológica en el Imperial College de Londres que culmina en 1991 con el robot "World First". Ese mismo año el robot PROBOT (Fig. 6) realizó una

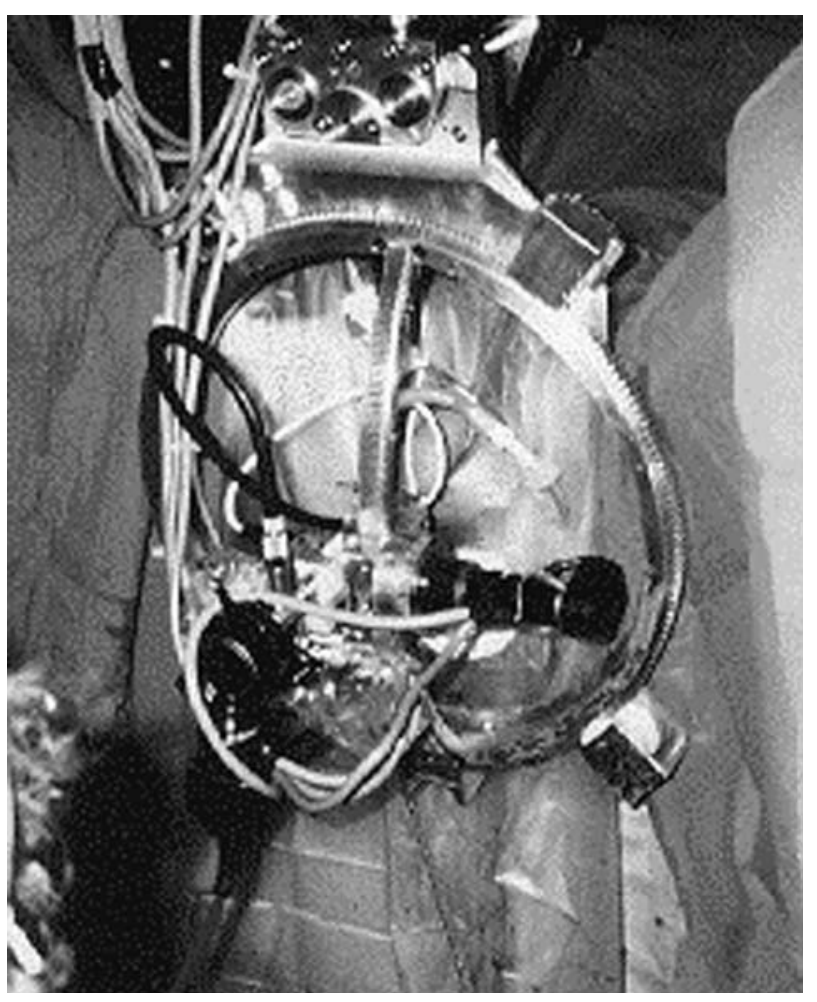

FIGURA 6. Aplicación clínica del robot PROBOT realizando una RTU de próstata. Tomado de http://www3.impe rial.ac.uk/mechatronicsinmedicine/projects/theprobot (Cortesia del Prof. Brian Davies, Imperial College of London, Departament of Mechanical Engineering). 
operación endoscopia de próstata, bajo la dirección del Dr. Simon Harris en el Guy's Hospital, London (Reino Unido) ${ }^{52}$ y abrió la posibilidad de realizar procedimientos robotizados endoscópicos aún en fase de I+D. También el robot puncionador renal PCNL fue desarrollado en el mismo instituto, siendo actualmente los miembros del Brady Urological Institute y la Johns Hopkins University, New York, USA ${ }^{53}$, quienes siguen con el desarrollo del robot. Su función consiste en realizar el acceso percutáneo al riñón mediante aguja de forma guiada por control remoto. El cirujano maneja un joystick a distancia.

En 1998 fue realizado el primer acceso renal percutáneo robotizado por telecirugía con PAKYRCM (Fig. 7), entre el Johns Hopkins Hospital, de Baltimore, MA, USA, y el Hospital Tor Vergara de la Universidad de Roma, Italia ${ }^{54}$. En el 2001 se publicaron los resultados de telecirugía con el sistema robotizado Mona de Intuitive Surgical: entre otros procedimientos se incluyeron 2 prostatectomías $^{55}$. El equipo de urólogos de la Universidad de California (Los Ángeles -UCLA-) realizó en enero del 2002 la primera cirugía abdominal con el robot Zeus, practicando una pieloplastia en una paciente de 22 años ${ }^{56}$.

La urología inició sus primeras experiencias con da Vinci en el 2001 con la prostatectomía radical, realizada por expertos cirujanos laparoscopistas $^{\text {57-59 }}$ mostrando buenos resultados hasta la actualidad $^{60}$. La primera nefrectomía laparoscópica se realiza en el 2001 con el objeto de extraer el

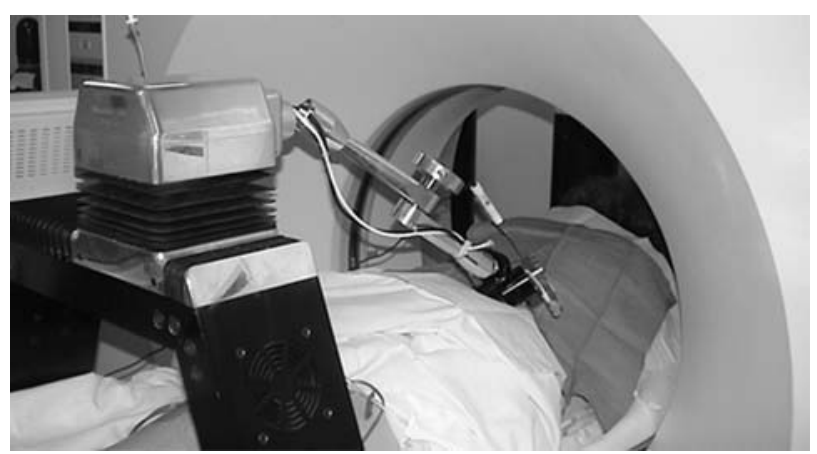

FIGURA 7. El robot PAKI-RCM realizando una nefrostomia por punción percutánea.

(Tomado de http://www.urology.jhu.edu/urobotics/) (Cortesia del Prof. Dan Stoianovici, Associate Professor of Urology and Mechanical Engineering, Brady Urological Institute, School of Medicine, Johns Hopkins University, New York, USA). riñón para trasplante ${ }^{61}$. Otras indicaciones, como la ureteropieloplastia ${ }^{62}$, cistectomía radical con neovejiga ${ }^{63}$ y nefrectomía radical ${ }^{64}$ y parcial $^{65}$ son asumidas posteriormente. La primera cirugía con el robot da Vinci en España fue una prostatectomía radical realizada por el Dr. Villavicencio en la Fundación Puigvert de Barcelona. En la actualidad ha sido presentado un nuevo modelo de este robot, el da Vinci S, que aporta avances como menor tamaño y más dinámica en los brazos.

\section{REFERENCIAS}

1. Sánchez Martín FM, Millán Rodríguez F, Salvador Bayarri J, Palou Redorta J, Rodríguez Escovar F, Esquena Fernández S, Villavicencio Mavrich H. Historia de la robótica: de Arquitas de Tarento al robot Da Vinci (Parte I). Actas Urol Esp. 2007;31(2):69-76. (en prensa).

2. Asimov I. Yo robot. Edhasa 1979. Barcelona.

3. http://en.wikipedia.org/wiki/I_Robot

4. Iñigo $\mathrm{R}$, Vidal $\mathrm{E}$ : Robots industriales manipuladores. Edicions UPC. Barcelona. 2002.

5. http://es.wikipedia.org/wiki/Prueba_de_Turing

6. http://en.wikipedia.org/wiki/Cybernetics

7. Angulo JM. Historia y evolución de la robótica. En Robótica práctica. Tecnología y aplicaciones. 13-14. Ed. Paraninfo. 5 ed. Madrid. 1999.

8. Fu KS, González RC, Lee GSG. Robótica. Control, detección, visión e inteligencia. Mc Graw-Hill. Madrid. 1990.

9. http://daniel-1491.tripod.com/id1.html, y http://www. geocities.com/Area51/Station/3173/versatran.html

10. http://en.wikipedia.org/wiki/Mechatronics

11. http://selena.sai.msu.ru/Home/Spacecrafts/Lunok hod1/lunokhodle.htm

12. Kahn ME, Roth B: The near-minimum time control of open loop articulated kinematic chains. J. Dyn. Syst. Measure. Control 1971;93(3):164-172.

13. Craig JJ. Introduction to robotics. Addison-Wesley $\mathrm{Pu}$ blishing Co. Reading (MA. USA). 1989.

14. Will PM, Grossman DD. An Experimental System for Computer Controlled Mechanical Assembly. IEEE Trans. Computers 1975;24(9):879-888.

15. Kelly R, Santibañez V. Control de movimiento de robots manipuladores. Pearson/Prentice Hall. Mexico. 2003.

16. http://news.bbc.co.uk/1/hi/in_depth/sci_tech/2001/ artificial_intelligence/1531432.stm

17. Shergill IS, Rao A, Arya M, Patel H, Gill IS. Nanotechnology: potential applications in urology. BJU Int. 2006;97 (2):219-220.

18. http://marsrovers.jpl.nasa.gov/home/index.html

19. http://www.hexapods.net/

20. http://www.ri.cmu.edu/projects/project_284.html

21. http://www.superrobotica.com/sr1_Robot.htm

22. http://www.irobot.com/sp.cfm?pageid=109

23. http://www.thinkbotics.com/ y http://home.golden.net/ $\sim \mathrm{kpwillia/}$

24. http: / /video.google.com/videoplay?docid=310330568 6876909316

25. http://robosavvy.com/KHR1Info/2

26. http://trueforce.com/Medical_Robotics/Medical_Robotics_Companies/aesop.htm 
27. http://xataka.com/2007/02/24-robot-asimo-bailando 28. http://www.irobot.com/sp.cfm?pageid=208

29. http://www.irobot.com/sp.cfm?pageid=310

30. http://www.irobot.com/sp.cfm?pageid=141

31. http://en.wikipedia.org/wiki/Wakamaru

32. http://daniel-1491.tripod.com/id1.html

33. Buckingham RA, Buckingham RO. Robots in operating theatres. Br Med J 1995; 311(7018):1479-1482.

34. http://en.wikipedia.org/wiki/Robotic_surgery\#History

35. http://www3.imperial.ac.uk/mechatronicsinmedicine

36. http://www.robodoc.com/eng/index.html

37. http://www3.imperial.ac.uk/mechatronicsinmedicine

38. http://www.robodoc.com/eng/neuromate.html

39. http://www.computermotion.com/aesop.html

40. http://trueforce.com/Medical_Robotics/Medical_Robotics_Companies/aesop.htm

41. Schurr MO, Buess G, Neisius B. Robotics and telemanipulation technologies for endoscopic surgery: a review of the ARTEMIS project. Surg Endosc. 2000;14(4):375-381.

42. Camarillo DB, Krummel TM, Salisbury Jr JK. Robotic technology in surgery: past, present, and future. Am J Surg. 2004; 188(4A Suppl):2S-15S.

43. Himpens J, Leman G, Cadiere GB: Telesurgical laparoscopic cholecystectomy. Surg Endosc 1998;12(8):1091.

44. Cadiere GB, Himpens J, Vertruyen M, Favretti F. The world's first obesity surgery performed by a surgeon at a distance. Obes Surg. 1999 Apr;9(2):206-209.

45. http: //www.fda.gov/fdac/features/2005/405_computer.html

46. Harris SJ, Arambula-Cosio F, Mei Q, Hibberd RD, Davies BL, Wickham JE et al. The Probot-an active robot for prostate resection. Proc Inst Mech Eng [H]. 1997;211(4): 317325.

47. http://www.hoise.com/vmw/01/articles/vmw/LV-VM10-01-20.html

48. http://www.hoise.com/vmw/01/articles/vmw/LV-VM08-01-25.html

49. Cadiere GB, Himpens J, Vertruyen M, Bruyns J, Fourtanier G. Nissen fundoplication done by remotely controlled robotic technique. Ann Chir 1999;53(2):137-141.

50. Melvin WS, Needleman BJ, Krause KR, Wolf RK, Michler RE, Ellison EC. Computer-assisted robotic Heller myotomy: initial case report. J Laparoendosc Adv Surg Tech A 2001;11(4):251-253.

51. Carpentier A, Loulmet D, Aupecle B, Berrebi A, Relland J. Computer-assisted cardiac surgery. Lancet 1999;353 (9150):379-380.

52. Harris SJ, Arambula-Cosio F, Mei Q, Hibberd RD, Davies BL, Wickham JE et al. Citado en http://www.urotoday.com/ prod/pdf/ reviews/BJU3_jan2005.pdf
53. http://www.Urology.jhu.edu/urobotics/

54. Challacombe BJ, Kavoussi LR, Dasgupta P. Trans-oceanic telerobotic surgery. BJU Int. 2003 Nov;92(7):678-680.

55. Cadiere GB, Himpens J, Germay O, Izizaw R, Degueldre M, Vandromme $\mathrm{J}$ et al. Feasibility of robotic laparoscopic surgery: 146 cases. World J Surg. 2001 Nov;25(11):1467-1477.

56. http://www.hoise.com/vmw/02/articles/vmw/LV-VM02-02-11.html

57. Abbou CC, Hoznek A, Salomon L, Olsson LE, Lobontiu A, Saint F, et al. Laparoscopic radical prostatectomy with a remote controlled robot. J Urol 2001;165(6 Pt 1):1964-1966.

58. Binder J, Kramer W. Robotically-assisted laparoscopic radical prostatectomy. BJU Int 2001;87(4):408-410.

59. Pasticier G, Rietbergen JB, Guillonneau B, Fromont G, Menon M, Vallancien G. Robotically assisted laparoscopic radical prostatectomy: feasibility study in men. Eur Urol. 2001;40(1):70-74.

60. Tewari A, Srivasatava A, Menon M. A prospective comparison of radical retropubic and robot-assisted prostatectomy: experience in one institution. BJU Int 2003;92(3): 205-210.

61. Horgan S, Vanuno D, Sileri P, Cicalese L, Benedetti E. Robotic-assisted laparoscopic donor nephrectomy for kidney transplantation. Transplantation 2002;73(9):1474-1479.

62. Hoznek A, Zaki SK, Samadi DB, Salomon L, Lobontiu A, Lang P, et al. Robotic assisted kidney transplantation: an initial experience. J Urol 2002;167(4):1604-1606.

63. Gettman MT, Neururer R, Bartsch G, Peschel R. AndersonHynes dismembered pyeloplasty performed using the da Vinci robotic system. Urology. 2002 Sep;60(3):509-513.

64. Beecken WD, Wolfram M, Engl T, Bentas W, Probst M, Blaheta R et al. Robotic-assisted laparoscopic radical cystectomy and intra-abdominal formation of an orthotopic ileal neobladder. Eur Urol. 2003 Sep;44(3):337-339.

65. Klingler DW, Hemstreet GP, Balaji KC. Feasibility of robotic radical nephrectomy-initial results of single-institution pilot study. Urology. 2005 Jun;65(6):1086-1089.

66. Olsen LH. Robotics in paediatric urology. J Ped Urol. 2006;2:40-45.

Correspondencia autor: Dr. FM. Sánchez-Martín Servicio de Urología. Fundació Puigvert.

Cartagena 340-350. 08025 Barcelona.

Tel.: 934169700

E-mail autor: fsanchez@fundacio-puigvert.es

Información artículo: Original - Historia de la Urología

Trabajo recibido: septiembre 2006

Trabajo aceptado: enero 2007 